\title{
Chewing-Gum as a Confounder for Intracranial Haemangiopericytoma Oral Metastasis
}

\author{
Maria Franco Echevarria ${ }^{1}$, Carlos Prol Teijeiro ${ }^{1 *}$, Alejandro Carrasco Gonzalez ${ }^{2}$ and \\ Josu Mendiola Arza ${ }^{3}$ \\ ${ }^{1}$ Oral \& Maxillofacial Surgery Department, Cruces University Hospital, Barakaldo, Spain \\ ${ }^{2}$ Neurosurgery Department, Cruces University Hospital, Barakaldo, Spain \\ ${ }^{3}$ Radiodiagnosis Department, Cruces University Hospital, Barakaldo, Spain
}

Received: May 2, 2017; Accepted: August 16, 2017; Published: September 18, 2017

"Corresponding author: Carlos Prol Teijeiro, c/Gernikako Arbola 30 6o izquierda, 48902, Barakaldo, Bizkaia, Spain, Tel: +0034 620112773, Email address: drprolteijeiro@gmail.com

\begin{abstract}
Intraoral foreign bodies are occasionally seen in scheduled or urgent X-ray examinations. They could cause potential misdiagnosis of oral pathology.

A clinical case of intracranial haemangiopericytoma, which is an infrequent malignant vascular tumour, is reported. The following-up shows a metastatic suspicious mouth image in a surveillance computed tomography (nodular, radiopaque, air bubbles surrounded). It turns to be a chewing-gum. A review of comestible intraoral foreign bodies radiological appearance is also carried out, focused on the soft chewable ones.

Additional imaging and distressing situations could be avoided with patient education and adherence to proper imaging protocols.
\end{abstract}

Key words: IOFB; Gum; Mouth Metastasis; Oral Abscess;

\section{Introduction}

Intraoral Foreign Bodies (IOFB) are frequently seen in scheduled or urgent $\mathrm{x}$-ray examinations of the head and neck. They could cause potential misdiagnosis of oral pathology. Extraction of any removable IOFB, including comestible or chewable ones, should be routinely requested before the patient undergoes the test.

Intracranial Haemangiopericytoma (HPC) is a rare malignant mesenchyma tumour, representing $0.4 \%$ of all primary central nervous system malignancies. HPC originates from abnormal pericytes of any capillary tissue. Clinically and radiologically, it resembles the meningioma, so the definitive diagnosis is histological, notably with staghorn vasculature and widespread reticulin as key features. Current management is surgery with adjuvant radiotherapy. Local recurrence rates are around 55\%, the majority between $1-5$ years after initial treatment. Metastasis rates are about 20\%, and mortality at ten years is $35 \%$ [1]. The extracranial head and neck location of primary or recurrent disease is also uncommon, at $16 \%$ of all HPC. Computed Tomography (CT) can show the following not specific findings: radiopaque soft tissue masses, cystic changes or calcifications in large and long evolution lesions [2].

We present a clinical case in which a comestible IOFB was initially confused with an oral metastasis. A review of comestible IOFB X-ray findings was also carried out, focusing on the soft chewable ones.

\section{Case report}

32-year-old male HPC patient was referred to our department of oral and maxillofacial surgery for management of an oral metastasis. Past history included $\beta$-lactam allergy and childhood asthma. The initial oncologic diagnosis was a left frontal HPC of $2 \mathrm{~cm}$, anaplastic grade III, which was previously managed with three neurosurgeries, namely a Subtotal Resection (STR) limited by blood loss, a wide local excision, and an excision of recurrence (Figure 1). The patient also received 50Gy of radiotherapy.

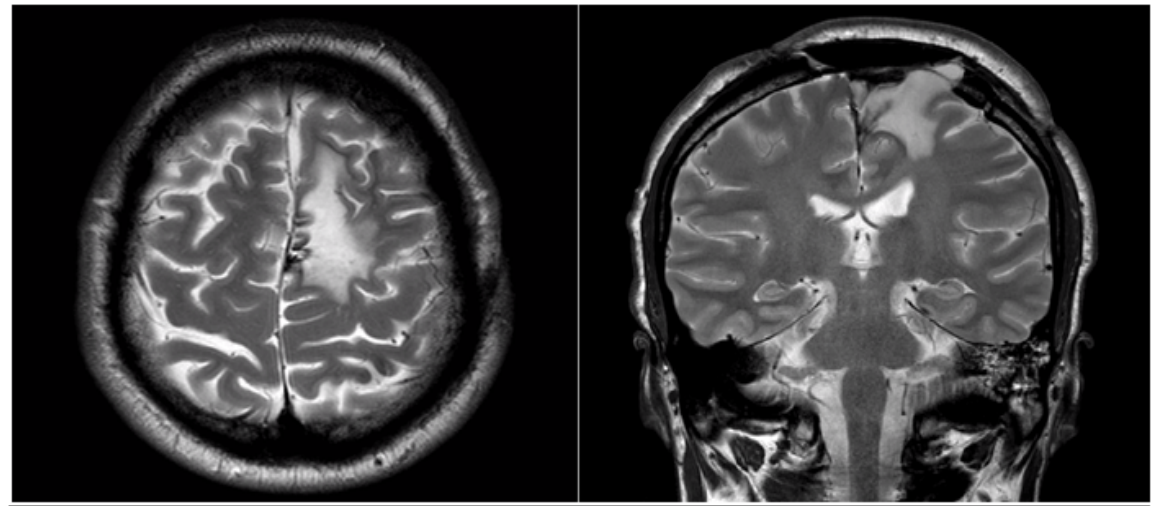

Figure 1: Cranial MRI T2 sequence after third surgery: Post surgical changes without tumour persistence data. Bifrontal and biparietal craniotomy covered with methyl-methacrylate prosthesis. Partial resection of left sagittal sinus and a left parasagittal encephalomalacic cavity. Axial, parasagittal and coronal slices.

Current symptoms and sequelae included left central deafness and partial seizures of the right hemibody, well controlled with high doses of levetiracetam. The physical examination was seemingly normal, with intact mucosa and unremarkable palpation of lymph nodes. On a surveillance CT five months after the last surgery, which prompted the referral to us, a solid heterogeneous lesion was found adjacent to the right mandibular ramus, ranging 310 Hounsfield Units (HU) of maximum attenuation (Figure 2). Questioning revealed that the patient was chewing gum during his CT. Repeat CT of the relevant area was normal (Figure 3).

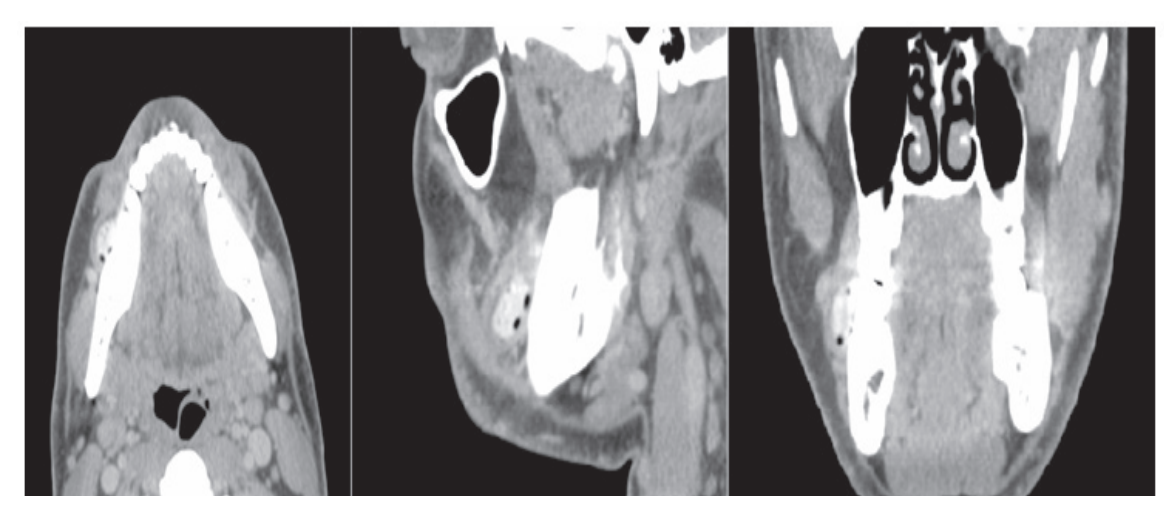

Figure 2: Mouth CT: Nodular, radiopaque, air bubbles surrounded, close to the right jaw ramus lesion. Axial, parasagittal and coronal slices. 


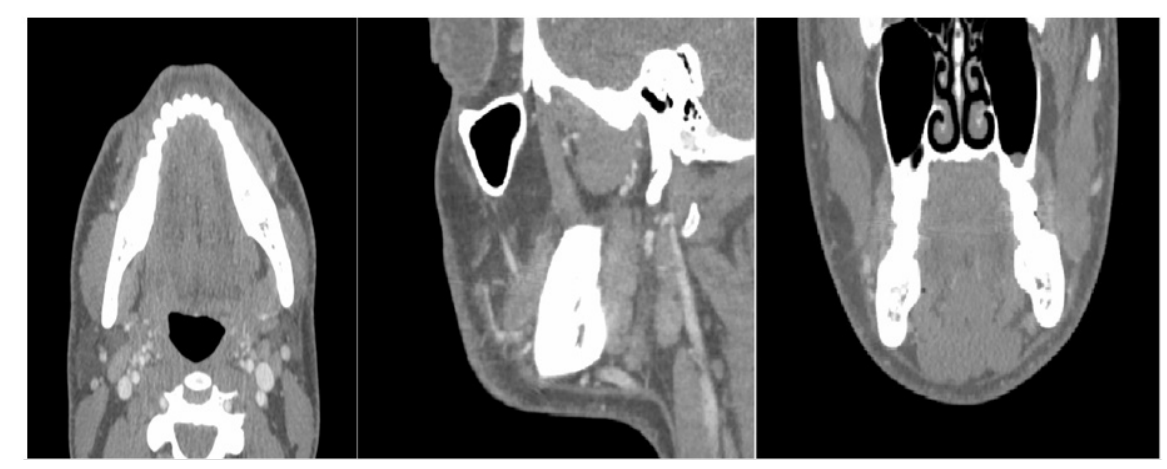

Figure 3: Mouth CT: Same slices of figure 2 without abnormalities.

\section{Discussion}

HPC patients need close oncologic surveillance. Upon finding an anomalous shaped hyperdense image on surveillance CT, as in our case, dangerous diagnoses such as a metastasis must be ruled out readily. This was especially important in our patient, due to the presence of early recurrence risk factors, notably a $\geq 6 \mathrm{~cm}$ sized primary lesion, non-skull base location, a first STR surgery, local recurrence, and tumour grade (anaplastic) [1].

Despite protocols for CT image acquisition in the Radiology Department, CT specific characteristics of different IOFB have been described [3, 4]. Soft IOFB, specifically chewing gum, are more radiodense than soft tissue (100-400 $\mathrm{HU}$ of maximum attenuation value), depending on their composition, whether it be gum base (elastomers, resins, fats, emulsifiers, waxes, anti-oxidants and fillers), sweetener, aromatic, or flavouring agents [5]. Furthermore, radiodensity is higher in vivo than in vitro samples generally. Also, IOFBs tend to show an oval or amorphous shape with air loculi around. The most common locations are mucosal recesses, and against the teeth, notably attached to the gingiva or hard palate $[3,4]$. Therefore, it is not uncommon to misinterpret IOFB with mucosal masses or abscesses, especially the softer and smaller they are.

In spite of the background, tissue consistency, shape, compatible location and radiopacity of our case, the acute finding, absence of clinical lesion, ranging $\mathrm{HU}$ and non-infiltrative radiological characteristics made us suspect something external could have disturbed the CT. Moreover, the heterogeneity and the airbubbles-surrounded peculiarity would be more compatible with an abscess than a metastatic lesion.
Other chewable IOFBs imaging characteristics have been documented in other locations too. Examples include calcifications in an abdominal x-ray and $\mathrm{CT}$, or increased fluorodeoxyglucose uptake of the masticatory muscles and surrounding inflammatory reaction in positron emission tomography [6-10].

\section{Conclusions}

Special attention must be taken in radiologic follow-up of malignancies, such as our case. It is important to be cognizant of IOFBs in the differential diagnosis of oral masses. Reducing their confounding presence on imaging should be feasible with patient education and adherence to proper imaging protocols. Thereby, additional x-ray examinations and distressing situations could be avoided.

\section{References}

1. Rutkowski MJ, Jian BJ, Bloch O, Chen C, Sughrue ME, Tihan T, et al. Intracranial Hemangiopericytoma: Clinical Experience and Treatment Considerations in a Modern Series of 40 Adult Patients. Cancer. 2012;118(6):1628-1636. doi: 10.1002/cncr.26411

2. Wushou A, Bai XF, Qi H, Xu Z, Zheng J, Li G. Haemangiopericytoma of the jaw. J Craniomaxillofac Surg. 2014;42(5):689-694.

3. McDermott M, Branstetter 4th BF, Escott EJ. What's in Your Mouth? The CT Appearance of comestible Intraoral Foreign Bodies. AJNR Am J Neuroradiol. 2008;29(8):1552-1555. doi: 10.3174/ajnr.A1127

4. Towbin AJ. The CT appearance of intraoral chewing gum. Pediatr Radiol. 2008;38(12):1350-1352. doi: 10.1007/s00247-008-0980-0

5. Imfeld T. Chewing Gum--Facts and Fiction: A Review of Gum-Chewing and Oral Health. Crit Rev Oral Biol Med. 1999;10(3):405-419

6. Ufberg JW, Lex J. Abdominal calcifications on unenhanced CT scan due to gummy bear ingestion. J Emerg Med. 2005;28(4):469-470.

7. Geller E, Smergel EM. Bubble gum simulating abdominal calcifications. Pediatr Radiol. 1992;22(4):298-299.

8. RimarY, Babich JP, Shaoul R. Chewing gum bezoar. Gastrointest Endosc. 2004;59(7):872.

9. Vock J, Juengling FD, Krause T, Wismeyer M. Muscular FDG uptake after chewing gum in a patient with Hodgkin disease. Clin Nucl Med. 2007;32(2):124-127.

10. Hall J, Movva A, Sridhar S, Chamberlain S. Education and imaging. Gastrointestinal: Adherent chewing gum in the colon causing a false positive PET scan. J Gastroenterol Hepatol. 2009;24(8):1472. doi: 10.1111/j.1440-1746.2009.05960.x 